Review Article

\title{
Cardiac Arrhythmias in Patients with End Stage Renal Disease (ESRD) on Hemodialysis; Recent Update and Brief Literature Review
}

\author{
Firas Ajam $^{1}$, Swapnil Patel ${ }^{1}$, Anas Alrefaee ${ }^{1}$, Dawn Calderon ${ }^{2}$, Mohammad A. Hossain ${ }^{1}$, Arif Asif ${ }^{1}$ \\ ${ }^{1}$ Department of Medicine, Jersey Shore University Medical Center, Hackensack Meridian Health, Neptune, USA \\ ${ }^{2}$ Department of Cardiology, Jersey Shore University Medical Center, Hackensack Meridian Health, Neptune, USA
}

Email address:

arif.asif@hackensackmeridian.org (A. Asif)

\section{To cite this article:}

Firas Ajam, Swapnil Patel, Anas Alrefaee, Dawn Calderon, Mohammad A. Hossain, Arif Asif. Cardiac Arrhythmias in Patients with End Stage Renal Disease (ESRD) on Hemodialysis; Recent Update and Brief Literature Review. American Journal of Internal Medicine.

Vol. 7, No. 1, 2019, pp. 22-26. doi: 10.11648/j.ajim.20190701.16

Received: February 15, 2019; Accepted: March 25, 2019; Published: April 22, 2019

\begin{abstract}
Cardiovascular diseases remains one of the leading causes of mortality worldwide, and causes more than one millions deaths annually in the United States alone. Patients with chronic kidney disease (CKD) are predisposed to different types of heart rhythm abnormalities including but not limited to atrial fibrillation, atrial flutter, ventricular tachycardia, and ventricular fibrillation. Both cardiovascular diseases and chronic kidney disease (CKD) may cause various variations in the cardiovascular system, autonomic nervous system, and various electrolytes abnormalities that open the door for the occurrence of multiple cardiac arrhythmias. Various pathophysiological mechanisms have been suggested in describing the correlation of cardiac arrhythmias in CKD patients. Despite the substantial progress in the management of chronic kidney disease, cardiovascular complications still highly dominant in CKD patient. Thus, reducing mortality from cardiovascular disease and sudden cardiac death in dialysis is a global health challenge. Management of cardiac arrhythmias includes the use of antiarrhythmic drugs, in addition to the use of catheter ablation and cardiac implantable electronic devices. The present review addresses the correlation between chronic kidney disease and different cardiac arrhythmias, the different pathophysiological mechanisms explaining the high risk of arrhythmias in CKD patients, the diagnostic tools used to identify arrhythmias, and the rule of subcutaneous defibrillators in preventing sudden cardiac death in dialysis patients.
\end{abstract}

Keywords: Cardiac Arrhythmia, Chronic Kidney Disease, Cardiovascular Disease, Cardiac Death

\section{Introduction}

The association between chronic kidney disease and cardiovascular disease has been an important topic of discussion in recent years. Cardiovascular diseases remains one of the leading cause of mortality worldwide, and causes more than one millions deaths in the United States alone [1]. Chronic Kidney Disease (CKD) is one of the most common comorbidity, seen in $10 \%$ of patients worldwide [2]. The most common causes of CKD are hypertension and diabetes mellitus [3]. The strong correlation between chronic kidney disease and cardiovascular disease, specifically dysrhythmias, has been well documented, with sudden cardiac death being the most common cause of mortality in patients with CKD with $25 \%$ of all-cause mortality [4]. Cardiac arrhythmia prevalence in patients with End Stage Renal Disease is known with one in three dialysis patients. Multiple pathophysiological mechanisms have been described in provoking arrhythmias in CKD patients [3].

We aim to perform a thorough literature review of different types of cardiac arrhythmias in CKD patients.

\section{Definitions}

Chronic Kidney disease (CKD) is defined as evidence of kidney damage or glomerular filtration rate (GFR) less than or equal to $60 \mathrm{ml} / \mathrm{min} / 1.73 \mathrm{~m}^{2}[5]$. 
Atrial fibrillation is an abnormal heart rhythm characterized by irregular quivering of the atria, usually starting as a brief episode of abnormal beating that becomes longer and more constant with time. It is considered a type of supraventricular tachycardia $[6,7]$

Atrial flutter is a common abnormal heart rhythm that originates in the atrium, characterized by fast, sudden onset (usually) regular heart rhythm and is classified as type of supraventricular tachycardia [8].

Ventricular tachycardia (VT) is an episode of three or more consecutive ventricular complexes, associated with a heart rate higher than $100 \mathrm{bpm}$. It is considered nonsustainied VT if it lasts less than 30 seconds and sustained VT if lasts more than 30 seconds. Ventricular tachycardia can be classified further into monomorphic and polymorphic based on the ORS complex pattern [8].

Ventricular fibrillation (VF) is a type of cardiac arrhythmia that characterized by disorganized electrical activity in the ventricles that makes the heart shaver instead of contract, leading to loss of consciousness and cardiac arrest [9].

Sudden cardiac arrest (SCA) is a sudden cessation of cardiac activity with hemodynamic collapse, it occurs within one hour from the beginning of the symptoms. In general population, arrhythmia accounts only for $5 \%$ to $10 \%$ of SCD in non-structural heart disease $[10,11]$.

\section{Epidemiology}

The relationship between $\mathrm{CKD}$ and increased risk for cardiovascular diseases is well established with almost $50 \%$ of CKD mortality is due to cardiac disease [12].

A study was done to assess the risk of cardiovascular disease and risk of death based on the degree of the eGFR. The risk of death was increasing as the eGFR decrease bellow $60 \ldots$ Patients with GFR between 15 to 29 had hazard ratio of 3.2, and rose to 5.9 in patients with GFR below 15 [13]. Another study showed similar results with profound increase in mortality as the eGFR fell below 60 [14].

Atrial Fibrillation is common in patients with CKD, with nearly $10-20 \%$ of patients with CKD having this arrhythmia [3]. A study that was done on CKD patients with ICD devices, showed higher rate of Anti-tachycardia Therapy Pacing (ATP) and Defibrillation in patients with CKD compared to other groups, suggesting higher occurrence of arrhythmias and shock events among CKD patient when compared to other groups [15].

\section{Pathophysiology}

Cardiovascular structural abnormalities are associated with increased risk of cardiac arrhythmias. A study showed that about $74 \%$ of CKD patients had signs of left ventricular hypertrophy (LVH) and 36\% had left ventricular dilation [3]. Cardiac remodeling in CKD patients has been linked to two main mechanisms: increased afterload and increased preload [12]. The increased afterload in CKD and ESRD is seen due to increased systemic arterial resistance, elevated arterial blood pressure and reduced large vessel compliance that will lead to myocardial thickening and concentric hypertrophy [12]. The Increased preload is caused by expansion of intravascular volume resulting in myocardial stretching. The development of anemia and the presence of atrioventricular fistulas might also promote cardiac remodeling in CKD patients. [12].

A study done on rat models, showed that those with CKD had longer action potential duration with more vulnerability to early after depolarization triggered activity, and ventricular arrhythmias [16].

A study discussing the non-traditional risk factors of uremia on the development of coronary artery disease in CKD patient was performed. Several uremia-related risk factors were found to play a role in the development of atherosclerosis in CKD patients. Carbamylated LDL (cLDL), a modified form of LDL, and metabolite of urea, has been found to induce endothelial cell injury and increase the expression of cell adhesion molecules and increase oxidative stress. P-cresylsulfate is another uremic factor that has been linked to endothelial dysfunction and major adverse cardiac events among patients with coronary artery disease. These factors play an important role in the development and the progression of coronary artery disease in CKD patients [17].

Coronary artery calcification (CAC) is an important predictor of cardiac events in both the general population and CKD patients. Higher level of CAC is found in ESRD patients, It can be explained by abnormal calcium and phosphorus metabolism [17].

Abnormal homocysteine metabolism and elevated homocysteine levels were consistently found in CKD patients on dialysis. High homocysteine levels can lead to oxidative stress and vascular endothelial injury, along with development of atherosclerosis [17].

Fibroblast growth factor-23 (FGF-23) is a hormone that promotes urinary phosphate excretion and regulated vitamin D metabolism. A low eGFR can potentially rise FGF-23. A study showed that high levels of circulating FGF-23 were associated with an increased risk of developing atrial fibrillation (AF) [18]. Endovascular wall thickening of the intramyocardial arteries in CKD patients can lead to reduction in the compensatory vasodilation capacity and subsequently restricting the ability of the heart to cope with the increased oxygen demand [19].

Cardiac fibrosis has an electrical sequelae that can be explained when fibrous tissue with high electrical resistance been interposed between cardio myocytes. This will cause a local delay in the spread of the action potential and lead to the development of reentry types of arrhythmia which is commonly seen in patient with CKD [20].

Non-renal patients who have survived sustained ventricular tachycardia were found to have high level of norepinephrine secreted from the sympathetic nerve endings. Patients with renal failure have elevated background sympathetic activity. This might explain their higher incidence of arrhythmias. [21]. 


\section{Diagnostic Tools}

\subsection{Electrocardiogram (EKG)}

Electrocardiogram (EKG) is considered one of the most affordable diagnostic screening tools that is primarily used to detect any kind of underlying cardiac abnormalities [5]. One or more EKG abnormalities have been noted in 50 to $86 \%$ of CKD patients in various studies [22].

The $\mathrm{P}$ wave parameters measured by 12-lead electrocardiogram (ECG) can be used as a noninvasive method to evaluate for left atrial enlargement. Increasing $\mathrm{P}$ wave dispersion and maximum $\mathrm{P}$ wave duration were independently associated with rapid decline of kidney function and progression to end stage renal disease on dialysis. Ultimately, measuring $\mathrm{P}$ wave parameters can be used as a screening tool help to identify patients at high risk for worse renal outcomes [23].

Another important electrical conduction abnormalities seen patients with CKD is Heart Rate Variability (HRV). HRV represents the variation of beat-to-beat intervals, and provides a noninvasive measurement for evaluating the autonomic nervous system (ANS). Reduced HRV in CKD patients represents an abnormal response of the autonomic nervous system, and is a significant risk factor for poor prognosis that include cardiac death, and development of coronary artery disease [24]. In a study conducted by Thomson BJ et al, a decrease in HRV was seen in nearly $75 \%$ of patients with CKD, emphasizing the importance for clinicians to be mindful of these markers [25].

\subsection{Echocardiography}

Echocardiography can be used to visualize changes in the heart. Echocardiographic changes in CKD patients include left atrial enlargement and left ventricular hypertrophy, both of these changes signal poor prognostic outcomes [26].

\subsection{Cardiac MRI}

Cardiac Magnetic Resonance Imaging (cMRI) is generally used as a noninvasive imaging modality to evaluate cardiac geometry and function. However, there are several limitations of cMRI in daily practice, such as high cost, nonportability, and little prognostic evidence of several cardiovascular diseases [26].

\section{Types of Arrhythmias in CKD}

\subsection{Supraventricular Tachycardia (SVT)}

Supraventricular tachycardia is common in patients with CKD [2]. Atrial Fibrillation is seen in $9-21 \%$ of CKD patients, with the number increasing to $13-27 \%$ of patients on long term hemodialysis [3]. Other types of SVT are found to be less common in dialysis patients [3]. Studies have suggests that two important factors which cause supraventricular tachycardia are age, and left atrial size [27, 28].

SVT is seen frequently immediately after dialysis, due dialysis associated ischemia and abnormal electrolyte levels [3]. Atrial Fibrillation seen in dialysis carries an extremely high mortality with a 5 year mortality rate of nearly $80 \%$ [3]. Chronic kidney disease and AF carry a number of shared risk factors and conditions that promote their incidence, possibly via systemic processes such as inflammation, oxidative stress, or fibrosis. Some evidence suggests that atrial fibrillation can also increases chronic kidney disease progression [2]. The occurrence of supraventricular tachycardia has been shown to carry high mortality however some study have shown that it is more indicative of underlying cardiovascular disease [3].

The treatment of supraventricular tachycardia in patients with CKD and End Stage Renal Disease (ESRD) is a challenge faced by clinicians. Dialysis patients pose certain challenges utilizing antiarrhythmic medications since CKD will affect the transport and the distribution of the medications as well as its metabolism. Due to altered pharmacokinetics, the efficacy and toxicities of medications should always been considered in patients. Other treatments such as

Catheter ablation can be complicated due to difficult vascular access in CKD patients [3].

\subsection{Ventricular Tachyarrhythmia and Sudden Cardiac Death}

The event rate of sudden cardiac death (SCD) is more common in CKD patients when compared to the general population, with 10 -fold increase once dialysis start. Studies showed that SCD is more common in hemodialysis patients in comparison to peritoneal dialysis during the first 6 months of starting dialysis [4]. The higher incidence of SCD among dialysis patients can be explained by the repolarization instability from the electrolytes abnormalities, poor homeostatic adjustment, sudden fluid removal, or by the ischemic effect of dialysis [3]. Bradycardia and asystole rather than ventricular tachycardia may be key causes of sudden death in hemodialysis patients. The rate of arrhythmias is higher during the first dialysis session and during the last 12 hours of each inter-dialytic interval [29].

Hemodialysis has been implicated as being a trigger for arrhythmias. In a large US cohort there was an increase in arrhythmia risk following the long inter-dialytic interval. Another retrospective study of 80 sudden death demonstrated a 1.7 fold increase in risk in the 12 hours prior to the end of the long inter-dialytic interval [30]. These data suggest that applying the same dialysis parameters without daily electrolytes tests may trigger fatal arrhythmias and can be a modifiable cause of sudden death [29]. Changing the dialysis session to be more extended or more frequent and increasing the use of resins might have a protective effect by reducing inter dialytic accumulation of fluid, electrolytes, and uremic toxins. An attempt to modify the dialysis prescription in response to more assessment of volume status and electrolytes may provide beneficial effect [29, 31]. 


\section{The Rule of Subcutaneous Defibrillators in Preventing Sudden Death in Dialysis Patients}

Different complications might happen with the traditional implantable cardioverter defibrillator (ICD). Preservation of the peripheral and the central veins in chronic kidney disease is important for successful hemodialysis. Central venous stenosis is frequently observed with transvenous leads and represents a serious condition as the central veins are important for blood return to the right atrium during dialysis. With the subcutaneous ICD, there is no transvenous leads and therefore no related damage to the central veins [32]. Endocardial cardiac rhythm device leads may lead to tricuspid regurgitation (TR) resulting in an increase pressure on the right side of the heart. This would add to the state of chronic volume load in hemodialysis patients leading to signs and symptoms of right sided heart failure [32]. The intravascular location of the leads serve a nidus for bacterial colonization [33]. Instead of utilizing the central veins, subcutaneous ICD does not have any leads, in this way all leads associated complications will be avoided [32]. Its utility is however limited to patients who do not require back up pacing.

\section{Conclusion}

Cardiac arrhythmias are common in CKD patients, with progressive increase in the incidence of sudden cardiac death with the loss of kidney function. Cardiovascular diseases is the leading cause of death in CKD patients and as such, its prevention and management is of utmost importance. The use of antiarrhythmic drugs require close monitoring for various risk factors. Non-invasive diagnostic tests including EKG, echocardiogram and cardiac MRI are suggested as the first step in investigating for underlying heart disease. The several benefits associated with use of subcutaneous defibrillators include the potential of it being highly valuable in this population. Given the high burden of cardiovascular diseases in CKD patients, further studies are required to improve the clinical outcome and the quality of life of CKD patients.

\section{Statement of Ethics}

The authors have no ethical conflicts to disclose

\section{Funding Sources}

This project was not supported by any grant or funding agencies

\section{Conflict of Interests}

All the authors do not have any possible conflicts of interest.

\section{References}

[1] Zheng, Z., Croft, J. B., Giles, W. H., \& Mensah, G. A. (2001). Sudden Cardiac Death in the United States, 1989 to 1998. Circulation, 104(18), 2158-2163. doi:10.1161/hc4301.098254.

[2] Wanner, C., Herzog, C. A., Turakhia, M. P., Blankestijn, P. J., Carrero, J., Clase, C. M., Winkelmayer, W. C. (2018). Chronic kidney disease and arrhythmias: Highlights from a Kidney Disease: Improving Global Outcomes (KDIGO) Controversies Conference. Kidney International, 94(2), 231-234. doi:10.1016/j.kint.2018.05.005.

[3] Roberts, P. R., \& Green, D. (2011). Arrhythmias in chronic kidney disease. Heart, 97(9), 766-773. doi:10.1136/hrt.2010.208587.

[4] US Renal Data System 2015 Annual Data Report: Epidemiology of Kidney Disease in the United States. (2016). American Journal of Kidney Diseases, 67(3). doi:10.1053/j.ajkd.2015.12.015.

[5] Bonato, F. O., \& Canziani, M. E. (2017). Ventricular arrhythmia in chronic kidney disease patients. Jornal Brasileiro De Nefrologia, 39(2). doi:10.5935/0101-2800.20170033.

[6] "Heart Disease Other Related Conditions". cdc.gov. September 3, 2014. Archived from the original on 14 February 2015. Retrieved 19 February 2015.

[7] Sodickson, A., \& Urman, R. D. (n.d.). Opioids. Essential Clinical Anesthesia Review, 133-136. doi:10.1017/cbo9781139584005.038.

[8] Sawhney, N. S., Anousheh, R., Chen, W., \& Feld, G. K. (2009). Diagnosis and Management of Typical Atrial Flutter. Cardiology Clinics, 27(1), 55-67. doi:10.1016/j.ccl.2008.09.010.

[9] "Types of Arrhythmia". NHLBI. July 1, 2011. Archived from the original on 7 June 2015. Retrieved 7 September 2016.

[10] "What Is Sudden Cardiac Arrest?". NHLBI. June 22, 2016. Archived from the original on 28 July 2016. Retrieved 16 August 2016.

[11] Chugh, S. S., Kelly, K. L., \& Titus, J. L. (2000). Sudden Cardiac Death With Apparently Normal Heart. Circulation, 102(6), 649-654. doi:10.1161/01.cir.102.6.649.

[12] Lullo, L. D., Gorini, A., Russo, D., Santoboni, A., \& Ronco, C. (2015). Left Ventricular Hypertrophy in Chronic Kidney Disease Patients: From Pathophysiology to Treatment. Cardiorenal Medicine, 5(4), 254-266. doi:10.1159/000435838.

[13] Go, A., Chertow, G., Fan, D., Mcculloch, C., \& Hsu, C. (2004). Chronic kidney disease and the risks of death, cardiovascular events, and hospitalization. ACC Current Journal Review, 13(12), 13. doi:10.1016/j.accreview.2004.11.016.

[14] Zachariah, D., Kalra, P. R., \& Roberts, P. R. (2014). Sudden cardiac death in end stage renal disease: Unlocking the mystery. Journal of Nephrology, 28(2), 133-141. Doi: 10.1007/s40620-014-0151-3.

[15] Kiuchi, M. G., Chen, S., \& Pürerfellner, H. (2017). Incidence of ventricular arrhythmic events in CKD patients with ICD. International Journal of Cardiology, 227, 312-317. doi:10.1016/j.ijcard.2016.11.094. 
[16] Hsueh, C., Chen, N. X., Lin, S., Chen, P., Gattone, V. H., Allen, M. R. Moe, S. M. (2014). Pathogenesis of Arrhythmias in a Model of CKD. Journal.

[17] Cai, Q., Mukku, V., \& Ahmad, M. (2014). Coronary Artery Disease in Patients with Chronic Kidney Disease: A Clinical Update. Current Cardiology Reviews, 9(4), 331-339. Doi: $10.2174 / 1573403 \times 10666140214122234$.

[18] Ryan, M. (2014). Faculty of 1000 evaluation for Fibroblast Growth Factor-23 and Incident Atrial Fibrillation: The Multi-Ethnic Study of Atherosclerosis (MESA) and the Cardiovascular Health Study (CHS). F1000 - Post-publication Peer Review of the Biomedical Literature. doi:10.3410/f.718447086.793497545.

[19] Dikow, R. (2004). Increased Infarct Size in Uremic Rats: Reduced Ischemia Tolerance? Journal of the American Society of Nephrology, 15(6), 1530-1536. doi:10.1097/01.asn.0000130154.42061.c6.

[20] Chen PS, Chou CC, Tan AY, Zhou S, Fishbein MC, Hwang C, Karagueuzian HS, Lin SF. The mechanisms of atrial fibrillation. J Cardiovasc Electrophysiol. 2006 Dec; 17 Suppl 3:S2-7.

[21] Ritz, E., \& Wanner, C. (2008). The Challenge of Sudden Death in Dialysis Patients. Clinical Journal of the American Society of Nephrology, 3(3), 920-929. doi:10.2215/cjn.04571007.

[22] Shafi, S., Saleem, M., Anjum, R., Abdullah, W., \& Shafi, T. (n.d.). ECG Abnormalities In Patients With chronic kidney disease. Retrieved from https://www.ncbi.nlm.nih.gov/pubmed/28712176.

[23] Huang, J., Wei, S., Chen, S., Chang, J., Hung, C., Su, H. Chen, H. (2014). P Wave Dispersion and Maximum P Wave Duration Are Associated with Renal Outcomes in Chronic Kidney Disease. PLOS ONE, 9(7). doi:10.1371/journal.pone.0101962.

[24] Huang, Y., Chang, Y., Chen, I., Yang, C., Leu, S., Su, H., Shiao, C. (2017). Correction: Heart rate variability during hemodialysis is an indicator for long-term vascular access survival in uremic patients. Plos One, 12(7). doi:10.1371/journal.pone.0181283.

[25] Thomson, B. J., Mcareavey, D., Neilson, J. M., Winney, R. J., \& Ewing, D. J. (1991). Heart rate variability and cardiac arrhythmias in patients with chronic renal failure. Clinical
Autonomic Research, 1(2), 131-133. doi:10.1007/bf01826209.

[26] The role of echocardiographic study in patients with chronic kidney disease. (2015, July 15). Retrieved from http://www.sciencedirect.com/science/article/pii/S092966461 5002326 .

[27] Korzets, A., Ori, Y., \& Herman, M. (2001, May). Serum potassium levels and atrial fibrillation in haemodialysis patients. Retrieved from https://www.ncbi.nlm.nih.gov/pubmed/11328938.

[28] Serum potassium levels and the risk of atrial fibrillation: The Rotterdam Study. (2013, August 23). Retrieved from https://www.sciencedirect.com/science/article/pii/S016752731 3015982.

[29] Roy-Chaudhury, P., Tumlin, J. A., Koplan, B. A., Costea, A. I., Kher, V., Williamson, D., Winkelmayer, W. C. (2018). Primary outcomes of the Monitoring in Dialysis Study indicate that clinically significant arrhythmias are common in hemodialysis patients and related to dialytic cycle. Kidney International, 93(4), 941-951. doi:10.1016/j.kint.2017.11.019.

[30] Bleyer, A., Hartman, J., Brannon, P., Reeves-Daniel, A., Satko, S., \& Russell, G. (2006). Characteristics of sudden death in hemodialysis patients. Kidney International, 69(12), 2268-2273. doi:10.1038/sj.ki.5000446.

[31] Bakris, G. L., Pitt, B., Weir, M. R., Freeman, M. W., Mayo, M. R., Garza, D. Bushinsky, D. A. (2015). Effect of Patiromer on Serum Potassium Level in Patients With Hyperkalemia and Diabetic Kidney Disease. Jama, 314(2), 151. doi:10.1001/jama.2015.7446.

[32] Vachharajani, T. J., Salman, L., Costanzo, E. J., Mehandru, S. K., Patel, M., Calderon, D. M. Asif, A. (2017). Subcutaneous defibrillators for dialysis patients. Hemodialysis International, 22(1), 4-8. doi:10.1111/hdi.12577.

[33] Carrillo, R. G., Garisto, J. D., Salman, L., Merrill, D., \& Asif, A. (2010). Contamination of Transvenous Pacemaker Leads Due to Tunneled Hemodialysis Catheter Infection: A Report of 2 Cases. American Journal of Kidney Diseases, 55(6), 1097-1101. doi:10.1053/j.ajkd.2010.01.009. 